\title{
Dynamics of visual perceptual decision-making in freely behaving mice
}

\author{
Wen-Kai You ${ }^{1,2}$ and Shreesh P. Mysore ${ }^{1,2 *}$ \\ ${ }^{1}$ Department of Psychological and Brain Sciences, Johns Hopkins University \\ ${ }^{2}$ The Solomon H. Snyder Department of Neuroscience, Johns Hopkins University \\ *Corresponding author: mysore@jhu.edu
}

\begin{abstract}
Studying the temporal dynamics of visual perceptual decision-making can offer key insights into cognitive processes contributing to it. Here, we investigated the time course as well as fundamental psychophysical constants governing visual perceptual decision-making in freely behaving mice performing 2-AFC orientation discrimination tasks. We did so by analyzing response accuracy against reaction time (i.e., conditional accuracy), and using drift diffusion modeling, in a series of experiments in which we varied target size, contrast, duration, and presence of a foil. Our results revealed two distinct stages in the time course of mouse visual decision-making - a 'sensory encoding' stage, in which conditional accuracy exhibits a classic tradeoff with response speed before asymptoting at a peak level, and a subsequent 'short term memory-dependent' stage exhibiting a classic asymptotic decay of performance following stimulus offset. We estimated the duration of visual sensory encoding as $\sim 300 \mathrm{~ms}$ across tasks, the lower bound of the duration of short-term memory as $\sim 1700 \mathrm{~ms}$, the briefest duration of visual stimulus input that is informative as $\sim 40 \mathrm{~ms}$, and the longest duration that benefits overall decision accuracy as $1000 \mathrm{~ms}$. Separately, by varying stimulus onset delay, we demonstrated that the conditional accuracy function and RT distribution, the two components of overall accuracy, can be independently modulated, and also estimated impulsivity of mice via an 'impulsivity index'. Our results reveal shared stages in mouse and human visual decision dynamics and establish a quantitative foundation for investigating the underlying neural circuit bases in mice.
\end{abstract}

\section{SIGNIFICANCE STATEMENT}

This study presents a quantitative breakdown of the time course of visual decision-making in mice during naturalistic behavior. It demonstrates parallel stages in mouse visual perceptual decision dynamics to those in humans, estimates their durations, and shows that mice are able to discriminate well under challenging visual conditions - with stimuli that are brief, low contrast, and small. This work sets the stage for investigating the neural bases of visual perceptual decision dynamics and their dysfunction in mice.

\section{INTRODUCTION}

Exploring the temporal dynamics of perceptual decisions from onset of the sensory input through the initiation of behavioral responses affords a key window into the underlying cognitive processes [1-3]. Investigations of such dynamics have led to rich insights into human visual decision-making[4, 5], and other species/modality [6-8]. They have revealed distinct stages in perceptual processing, their timing, and their interactions, and have highlighted the importance of conditional accuracy analysis (over just conventional reaction time analyses) for investigating dynamics of perception and decision-making [9-11]. Conducting such investigations in a genetically tractable animal model can additionally facilitate the subsequent unpacking of the mechanistic basis of different stages in perceptual dynamics. However, despite the recent rise in the use of the laboratory mouse for the study of the visual system [12-14] and of visually guided decision-making [15-25], the temporal dynamics of visual perceptual decisions represents a significant gap in mouse visual psychophysics [26, 27]. 
In this study, we adapted approaches from human psychophysical studies to investigate the dynamics of visual decision-making in freely behaving mice. In a series of experiments involving touchscreen-based $[24,28]$ 2-alternative forced choice (2-AFC) orientation discrimination tasks, we investigated the effect of stimulus size, contrast, duration, delay, and the presence of a competing foil on mouse decision performance (accuracy, sensitivity and RT), and importantly, on the conditional accuracy function. We identified two distinct stages in the time-course of mouse visual decision-making within a trial, as has been reported in humans [29-36]. In the first 'sensory encoding' stage [29-32], response accuracy exhibited a classic tradeoff with response speed, and asymptoted to a peak level. In the next stage, response accuracy did not exhibit such a tradeoff, but instead, declined following stimulus offset, exhibiting a classic asymptotic decay to chance performance consistent with a short-term memory (STM)-dependent process [33-36]. Combining these results with those from drift diffusion modeling [37] allowed us to estimate fundamental psychophysical constants in mouse perceptual decision-making: the time needed by mice to complete visual sensory encoding, the duration for which their short term memory can intrinsically support discrimination behavior after stimulus input is removed, the shortest visual stimulus duration that is informative, and the longest stimulus duration beyond which no additional benefit in overall decision accuracy is seen. Additionally, by varying stimulus onset delay, we demonstrated that the two components of accuracy, namely, the conditional accuracy function and the RT distribution, can be independently modulated by task parameters. This last experiment also allowed a quantitative estimation of impulsivity of mice via a novel 'impulsivity index'. Together, this study reveals parallels between mouse and human visual decision dynamics, despite differences in their sensory apparatuses, and enable investigations into the neural circuit underpinnings of the timecourse of perceptual decision-making in mice.

70

71

72

73

74

75

76

77

78

79

80

81

82

\section{METHODS}

Animals. Thirty-three C57B16/J male mice (Jackson Labs) were housed in a temperature $(\sim 75 \mathrm{~F})$ and humidity $(\sim 55 \%)$ controlled facility on a $12: 12 \mathrm{~h}$ light:dark cycle; ZT0=7 am. All procedures followed the NIH guidelines and were approved by the [Author University] Animal Care and Use Committee (ACUC). Animals were allowed to acclimate for at least one week, with ad libitum access to food and water before water regulation was initiated per previously published procedures [38]. Briefly, mice were individually housed (for monitoring and control of daily water intake of each identified animal), and administered $1 \mathrm{~mL}$ water per day to taper their body weight down, over the course of 5-7 days, to $80-85 \%$ of each animal's free-feeding baseline weight. During behavioral training/testing, the primary source of water for mice was as a reinforcer for correct performance: $10 \mu \mathrm{L}$ of water was provided for every correct response. Experiments were all carried out in the light phase.

Apparatus. Behavioral training and testing were performed in soundproof operant chambers equipped with a touchscreen (Med Associates Inc.), a custom-built reward port (fluid well), infrared video cameras, a house light and a magazine light above the reward port. The reward port was located at the opposite wall of the chamber relative to the touchscreen (Fig. 1A, 1-1A). Mice were placed within a clear plexiglass tube ( $5 \mathrm{~cm}$ diameter) that connects the touchscreen and the reward port. A thin plexiglass mask ( $3 \mathrm{~mm}$ thickness) was placed $3 \mathrm{~mm}$ in front of the touchscreen with three apertures $(1 \mathrm{~cm}$ diameter) through which mouse was allowed to interact with the screen via nose-touch. The 'left' and 'right' apertures were placed $3 \mathrm{~cm}$ apart (center-to-center) along the base of the triangle, and a 'central' aperture, at the apex of the triangle, was 1.5 $\mathrm{cm}$ below the midpoint of the base. All experimental procedures were executed using control software (Klimbic, Med-Associates).

Visual stimuli. Visual stimuli were bright objects on the dark background (luminance $=1.32 \mathrm{~cd} / \mathrm{m}^{2}$ ). A small cross $\left(60 \times 60\right.$ pixels; luminance $\left.=130 \mathrm{~cd} / \mathrm{m}^{2}\right)$ was presented in the central aperture and had to be touched to initiate each trial. Oriented gratings (horizontal or vertical) were generated using a square wave, with fixed spatial frequency ( 24 pixels/cycle) known to be effective for mice to discriminate [17]. The dark phase of the grating was black as the background (luminance, $\mathrm{L}_{\text {dark }}=1.32 \mathrm{~cd} / \mathrm{m}^{2}$ ), and the bright phase was varied between $1.73 \mathrm{~cd} / \mathrm{m}^{2}$ and $130 \mathrm{~cd} / \mathrm{m}^{2}$ depending on the tasks (see below). The contrast of grating 
stimulus was calculated as the Michelson contrast $=\left(\right.$ luminance $_{\text {bright }}-$ luminance $\left._{\text {dark}}\right) /$ (luminance $_{\text {bright }}+$ luminance $\left._{\text {dark }}\right) * 100$; The size of the stimulus was also varied depending on the task, ranging from 60 pixels x 60 pixels to 156 pixels x 156 pixels, which subtended 25-65 visual degrees at a viewing distance of $2 \mathrm{~cm}$ from the screen (Fig. 1-1A).

Experimental procedure and behavioral training. Each mouse was run for one 30 min behavioral session per day, with each session yielding 80-180 trials. Each trial in a session was initiated by the mouse touching the zeroing cross.Upon trial initiation, the cross vanished, and the visual stimulus (or stimuli) were immediately presented (except in the delay task), for a duration of 0.1-3s depending on the task (see below).Mice were trained to report the orientation of target grating, by nose-touching the correct response aperture (vertical $\rightarrow$ left; horizontal $\rightarrow$ right). A correct response triggered a tone $(600 \mathrm{~Hz}, 1 \mathrm{sec})$, the magazine light turning on, and the delivery of $10 \mu \mathrm{L}$ of water. When mice turned to consumed the reward, their head entry into the reward port was detected by an infrared sensor which caused the zeroing cross (for the next trial) to be presented again. An incorrect response triggered a 5-s timeout, during which the house light and the magazine light were both on and zeroing cross was unavailable for the next trial to be initiated. A failure to respond within $3 \mathrm{~s}$ (starting stimulus presentation) resulted in a trial reset: the stimulus vanished and the zeroing cross was presented immediately (without a timeout penalty), to allow initiation of the next trial. Well-trained animals failed to respond on fewer than $5 \%$ of the total number of trials, and there were no systematic differences in the proportion of such missed trials between different conditions. Within each daily 30-minute behavioral session, mice consumed approximately $1 \mathrm{~mL}$ of water. If a mouse failed to collect enough water from the behavioral session, they were provided with a water supplement using a small plastic dish in their home cage.

Single-stimulus discrimination task. Upon trial initiation, a single grating stimulus (i.e., the 'target') was presented above the central aperture, at the same horizontal level as the left and right apertures, and mice were required to report its orientation with the appropriate nose-touch (Fig. 1B). When stimulus size and contrast were manipulated (Fig. 1, and 2), three different sizes were tested: 60x60, 84x84, 108x108 (pixels $\mathrm{x}$ pixels). Of each size, seven different levels of contrast were tested: $20 \%, 32 \%, 54 \%, 70 \%, 85 \%, 93 \%$, $98 \%$. Trials with different stimulus contrasts at a particular size were interleaved randomly throughout a session, while trials with different stimulus sizes were examined on different days. Data were recorded from a total of 18 sessions (days). When stimulus size was manipulated independently (Fig. S1J), full-contrast (98\%) gratings of different sizes were tested: 60x60, 84x84, 108x108, 132x132, 156x156 (pixels x pixels). Trials with different stimulus sizes were interleaved randomly throughout a session, and data were recorded from a total of five sessions (days). When the stimulus duration was manipulated (Fig. 3), the contrast (98\%) and size $(60 x 60)$ of the grating were fixed, and eleven different stimulus durations were tested: 100, 200, $300,400,500,600,800,1000,1500,2000,3000 \mathrm{~ms}$. The stimulus duration was fixed for a given day, and across days, was varied in a descending sequence from $3000 \mathrm{~ms}$ to $100 \mathrm{~ms}$. Data were recorded from a total of 21 sessions. When the stimulus onset delay was manipulated (Fig. 5), the contrast (98\%), size (60x60), and duration $(600 \mathrm{~ms})$ of the grating were fixed. Three different delays were tested: 0,100 , and $200 \mathrm{~ms}$. The delay duration was fixed for a given day, and varied in an ascending sequence from $0 \mathrm{~ms}$ to $200 \mathrm{~ms}$. Data were recorded from a total of 7 sessions (days).

Flanker task. Upon trial initiation, either one stimulus ('target', $60 \mathrm{x} 60$ pixels, contrast=88\%) was presented at the lower location, or two stimuli were presented simultaneously, with the target at the lower location and a second 'flanker' at the upper location (Fig.4A). Flankers were of the same size (60x60) and spatial frequency ( $24 \mathrm{pixel} / \mathrm{cycle}$ ) as the target, but of contrast in 8 different levels: $13 \%, 27 \%, 41 \%, 58 \%$, $74 \%, 88 \%, 94 \%, 98 \%$. The orientation of the flanker was either identical to that of the target ('congruent trial') or orthogonal to that of the target ('incongruent trial'). The stimulus (stimuli) was (were) presented for a duration of $1 \mathrm{~s}$, and mice were required to report orientation of the target grating with the appropriate nose-touch (within 3s). All types of trials (no flanker, congruent, incongruent) and flanker contrasts were interleaved randomly within each daily session. Data from this experiment have been reported previously 
[24], and were re-analyzed here using different analyses, after collapsing trials across all the contrasts of the flanker.

Subject inclusion/exclusion. 25 out of 33 mice passed the inclusion threshold of response accuracy $>70 \%$ in single stimulus discrimination task. Of these, different subsets of mice were used in different tasks. For mice involved in more than one experiment, they were well rested for 3-8 weeks with food and water ad libitum between experiments. Before the start of each experiment, all mice were given a few days of practice session to ensure that they remembered/re-learned the association between the orientation of single target and the appropriate nose-touch.

Trial inclusion/exclusion. Mice were observed to become less engaged in the task towards the end of a behavioral session, when they had received a sizeable proportion of their daily water intake. This was reflected in their behavioral metrics: they tended to wait longer to initiate the next trial, and their performance deteriorated. We identified and excluded such trials following a published procedure [24], in order to minimize confounds arising from loss of motivation towards the end of sessions. Briefly, we pooled data across all mice and all sessions, treating them as coming from one session of a single 'mouse'. We then binned the data by trial number within the session, computed the discrimination performance in each bin (\% correct), and plotted it as a function of trial number within session (Fig. 1-1C, 3-1, 5-1A). Using a bootstrapping approach, we computed the $95 \%$ confidence interval for this value. We used the following exclusion criterion: Trials $\mathrm{q}$ and above were dropped if the $\mathrm{q}^{\text {th }}$ trial was the first trial at which at least one of the following two conditions was satisfied: (a) the performance was statistically indistinguishable from chance on the $\mathrm{q}^{\text {th }}$ trial and for the majority (3/5) of the next 5 trials (including the $\mathrm{q}^{\text {th }}$ ), (b) the number of observations in $\mathrm{q}^{\text {th }}$ trial was below $25 \%$ of the maximum possible number of observations for each trial (mice*sessions), thereby signaling substantially reduced statistical power available to reliably compare performance to chance. The plots of performance as a function of trial number, and number of observations as a function of trial number for the different tasks in this study are shown in Figs. 1-1C, 3-1, 5-1A, along with the identified cut-off trial numbers $(q)$.

Behavioral measurements: Response accuracy (\% correct) was calculated as the number of correct trials divided by the total number of trials responded (correct plus incorrect). Reaction time (RT) was defined as the time between the start of stimulus presentation and response nose-touch, both detected by the touchscreen. In the experiment involving stimulus onset delays (Fig. 5A), RT was computed with respect to trial initiation (for clarity; as opposed to from stimulus onset), and denoted 'RT w.r.to trial initiation'.

Signal detection analysis (sensitivity and criterion). In the framework of signal detection theory, we assigned the correct vertical trials as 'hits', incorrect vertical trials as 'misses', correct horizontal trials as 'correct rejections' and incorrect horizontal trials as 'false alarms', and calculated the perceptual sensitivity (d') and criterion (c) accordingly [39] (Fig. S1B). Because of the inherent symmetry in 2-AFC tasks, this calculation was independent of which grating orientation - vertical or horizontal - was assigned as 'signal' and which as 'noise'. Consequently, a positive value of c caused poor performance just as much as the corresponding negative value, and therefore, we quantified the absolute value of $\mathrm{c}(|\mathrm{c}|)$ as the relevant metric of decision criterion.

Drift diffusion modeling of RT distributions. To shed light on potential mechanisms underlying observed RT distributions, we applied the drift-diffusion model to our RT data [40, 41]. This model hypothesizes that a subject ('decision maker') collects information from the sensory stimulus via sequential sampling, causing sensory evidence to accrue for or against a particular option (usually binary) while viewing the stimulus. A decision is to be made when the accumulating evidence reaches an internal threshold of the subject. This process of evidence accumulation, together with the processes of sensory encoding and motor execution, as well as threshold crossing, determine the RT observed on each trial (Fig. 1-1D). 
We used a standard version of the model that consists of four independent variables [37, 42]: (1) the drift rate, (2) the boundary separation, (3) the starting point, and a (4) non-decisional constant ( $\left.t_{\text {delay }}\right)$, which accounts for the time spent in sensory encoding and motor execution. In the case of our tasks, there was no reason for the drift rate to be different between vertical versus horizontal gratings, and therefore, we merged both type of trials (trials with a horizontal target grating and trials with a vertical target grating). We treated 'correct' response and 'incorrect' response as the two binary options, and fit the diffusion model to the RT distributions of correct versus incorrect trials using the fast-dm-30 toolbox with the maximum likelihood option to gain estimates of those four parameters for each individual mouse [40].

Conditional accuracy analysis. In order to get the full distribution of RT, trials from all mice were pooled together and treated as if they were from one single mouse. Pooled trials were then sorted by their RT, and then binned by RT into $50 \mathrm{~ms}, 100 \mathrm{~ms}$ or $200 \mathrm{~ms}$ bins, depending on the total number of trials available in each experiment. Conditional accuracy was calculated as the number of correct trials divided by the total number of trials for each RT bin.

Conditional accuracy function (CAF). To quantitatively describe the relationship between the conditional accuracy and RT, we fitted the plot of discrimination accuracy against (binned) RT with different functions (the CAF, see below) using a nonlinear least square method.

For RT bins aligned to stimulus onset (Fig. 2, 4C, 5C), we fit the data using an asymptotic function: accuracy $\left.=\lambda\left(1-\mathrm{e}^{-\operatorname{\gamma enc}(\mathrm{RT}-\delta)}\right)\right)$. Three key metrics were defined for the sensory encoding phase for the use in subsequent comparisons between trial conditions: (1) peak conditional accuracy $\left(a_{\text {peak }}\right)$, the maximal level of accuracy that the CAF reaches within the range of RT; (2) the timepoint at which the conditional accuracy reaches its maximal ( $\left.\mathrm{t}_{\text {peak }}\right)$. We defined it as the time point when the ascending CAF reaches $a_{\text {peak }} * 0.95$; and (3) the timepoint at which the conditional accuracy just exceeds chance level of performance $\left(\mathrm{t}_{50}\right)$. We defined it as the time point when the ascending CAF crosses $52.5 \%$ (i.e., $50 \% * 1.05$ ). Note that $t_{\text {peak }}$ and $t_{50}$ are influenced by the slope parameter, $\gamma_{e n c}$, and the temporal offset at chance performance, $\delta$.

For RT bins aligned to stimulus offset (Fig. 3D, 4E, 5D), we fit the data using a sigmoid function: accuracy $=\lambda\left[1 /\left(1+\mathrm{e}^{-\beta \operatorname{dec}(\mathrm{RT}-\tau)}\right)\right]+50$ to quantify the time course of performance decay. Two key metrics were defined for this VSTM phase for the use in subsequent comparisons between trial conditions: (1) the first time point at which the conditional accuracy drops from its maximum $\left(\mathrm{t}_{\text {decay }}\right)$. We defined it as the time point when the descending CAF crosses $a_{\text {peak }} * 0.95$; and (2) the first timepoint at which the conditional accuracy drops to a level indistinguishable from the chance $\left(t_{\text {chance }}\right)$. We defined it as the timepoint when the descending CAF crosses $52.5 \%$. In (rare) cases when the CAF never went below $52.5 \%$, $t_{\text {chance }}$ was set to be $3000 \mathrm{~ms}$. Note that $t_{\text {decay }}$ and $t_{\text {chance }}$ are influenced by the slope parameter, $\beta_{\text {dec }}$, and $\tau$.

The confidence interval of the CAF and each metric were estimated by bootstrapping: the same number of trials were resampled from the raw data randomly with replacement, and were then processed following the same steps as described above to get repeated estimates of the CAF and corresponding metrics. Such resampling was repeated 1000 times to estimate the dispersion of each metric. Plots of the estimated value of each metric show the mean \pm std of the bootstrapped distribution of estimates (Fig. 2CE, 3D, 4DF, 5$1 \mathrm{BC})$.

Statistical tests. All analyses and statistical tests were performed in MATLAB. For single-stimulus experiments in which only one stimulus parameter was systemically varied, one-way ANOVA was applied to examine the effect of the manipulating the single factor (duration and delay, Fig. 3ABC, 5AB, 1-1J). For experiments that involved changing both stimulus size and contrast (Fig. 1CDE, 1-1E-H), two-way ANOVA was applied to examine the effect of each factor, as well as their interaction.

For the flanker task, the Wilcoxon signed-rank test was used to examine if the group performance was different between trial types (Fig. 4B).

The Pearson correlation coefficient and associated p-value were calculated for paired data (Fig. 3ABC, $5 \mathrm{AB}, 1-1 \mathrm{I})$ using corrcoef function in MATLAB; correlation was also used to evaluate whether there was a trend in the data that did not show significance with ANOVA. 
For the metrics associated with CAF, permutation tests were used to determine if the estimated values of each metric were different between experimental conditions (Fig.2CE, 4DF, 5-1BC). Specifically, trials from both conditions were pooled together (unlabeled), and randomly re-assigned into two groups. The best-fit CAF and associated metrics were then calculated for each group, and so was the difference of metrics between groups. Following 1000 repetitions, the resulting distribution of the difference of metrics between groups was obtained under the null hypothesis that the data from the two conditions were indistinguishable (i.e., from the same distribution). The real, observed difference of metrics obtained from the two experimental conditions (for instance, $\Delta \mathrm{a}_{\text {peak }}$ from low-contrast vs. high contrast conditions) was compared against the null distribution to compute the corresponding p-value.

Correction for multiple comparisons was performed where necessary using the Holm-Bonferroni test (HB test) for multiple comparisons.

\section{RESULTS}

In this study, freely behaving mice were trained to perform 2-AFC orientation discrimination in a touchscreen-based setup [24, 28](Methods). Briefly, mice were placed in a plexiglass tube within a soundproof operant chamber equipped with a touch-sensitive screen at one wall and a reward well at the opposite wall (Fig. 1A, S1A). A plexiglass sheet with three holes was placed in front of the touchscreen the holes corresponded to the locations at which mice were allowed to interact with the screen by a nosetouch (Fig. 1A). All trials began with a nose-touch on a bright zeroing-cross presented within the lower central hole (Fig. 1B). Immediately following nose-touch, an oriented grating (target; bright stimulus on a dark background) was presented at the center of the screen. Mice were rewarded if they responded to the orientation of the target with an appropriate nose-touch: vertical (horizontal) grating $\rightarrow$ touch within upper left (upper right) hole. Behavioral data were collected from daily sessions that lasted 30 minutes for each mouse.
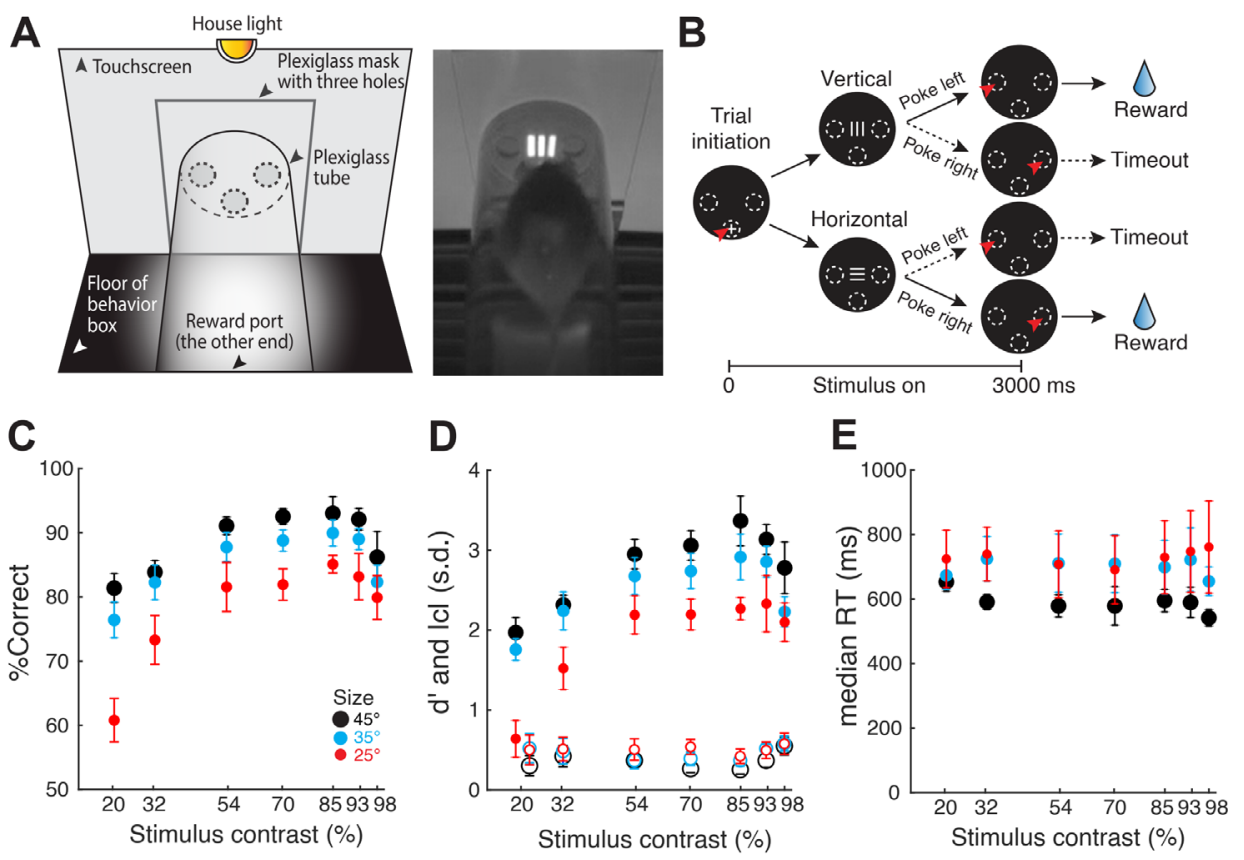

Figure 1. Stimulus contrast and size modulate orientation discrimination performance in freely behaving mice. (A) Left: Schematic of touchscreen-based experimental setup showing key components. Right: Snapshot of freely behaving mouse facing a visual stimulus on the touchscreen. (B) Schematic of 2-AFC task design. Black discs: Screenshots of touchscreen with visual stimuli; dashed ovals: locations of holes through which mice can interact with touchscreen; white '+': zeroing cross presented within central response hole at start of each trial; red arrowhead: nosetouch by mouse. Shown also are vertical or horizontal grating stimuli, and reinforcement (water)/punishment (timeout) schedule. Bottom: Trial timeline. $0 \mathrm{~ms}$ corresponds to the instant at which the mouse touches the zeroing cross (trial 
initiation). Immediately following this, the target grating was presented and stayed on for $3 \mathrm{~s}$, or until the mouse responded, whichever came first. Vertical and horizontal targets were interleaved randomly. (C) Psychometric plots of discrimination accuracy against stimulus contrast (Michelson contrast: (luminance bright-luminance $_{\text {dark}}$ )/ (luminance $_{\text {bright }}+$ luminance dark) $^{*} 100$; Methods). Different colors correspond to different target sizes. Data: mean \pm s.e.m; $\mathrm{n}=8$ mice. 2 -way ANOVA, $\mathrm{p}<0.001$ (contrast), $\mathrm{p}<0.001$ (size), $\mathrm{p}=0.498$ (interaction). (D) Plot of discrimination sensitivity (d', filled data) and critera ( $|\mathrm{c}|$, hollow data) against stimulus contrast. 2-way ANOVA, d': $\mathrm{p}<0.001$ (contrast), $\mathrm{p}<0.001$ (size), $\mathrm{p}=0.899$ (interaction); $|\mathrm{c}|: \mathrm{p}=0.374$ (contrast), $\mathrm{p}=0.056$ (size), $\mathrm{p}=0.998$ (interaction). The $\mathrm{d}^{\prime}$ and $|\mathrm{c}|$ were plotted with a slight offset at contrast $=20 \%$ to avoid overlapping. (E) Plot of median reaction time (RT) against stimulus contrast. 2-way ANOVA, $\mathrm{p}=0.99$ (contrast), $\mathrm{p}=0.004$ (size), $\mathrm{p}=1$ (interaction).

\section{See also Fig. 1-1.}

\section{Stimulus size and contrast modulate mouse discrimination performance}

We first examined the effect of target size and target contrast on the decision performance of mice in the orientation discrimination task. Here, the target grating was presented for up to 3 seconds after trial initiation (Fig.1B; Methods), and its size and contrast were systematically varied; the spatial frequency was fixed at 0.1 cycles/degree (24 pixels/cycle) $[16,17]$ (Methods). Mice were allowed to respond at any time during stimulus presentation, and the stimulus was terminated automatically upon response.

We found that both the target contrast and size significantly modulated discrimination accuracy (Fig. 1C, 2 -way ANOVA, main effect of contrast, $p<0.001$; main effect of size, $p<0.001$; interaction, $p=0.498$ ). The improvements in response accuracy observed with increasing target contrast as well as target size were accompanied by improvements in perceptual sensitivity (Fig. 1D, filled symbols; 2-way ANOVA, main effect of contrast, $p<0.001$; main effect of size, $p<0.001$; interaction, $p=0.899$ ), but no detectable change in decision criterion (Fig. 1D, open symbols). These results revealed that mice discriminated target orientation better than chance even at the lowest contrast $(20 \%)$ and size $\left(25^{\circ}\right)$ tested (Fig. 1C; red dot at the left lower corner, $\mathrm{p}=0.039$, Wilcoxon signed rank test). Additionally, at this smallest target size $\left(25^{\circ}\right)$, mice could discriminate with $>80 \%$ accuracy and d' $>2$ s.d. for most of the stimulus contrasts ( $\geq 54$; Fig. $1 \mathrm{CD}$, red data).

The effect of these parameters on median response times (RTs) was less pronounced. Target size, but not contrast, modulated reaction times (RTs) (Fig.1E, two-way ANOVA; main effect of size, $\mathrm{p}=0.004$; main effect of contrast, $\mathrm{p}=0.998$; interaction, $\mathrm{p}=1)$.

We also analyzed the full RT distributions using the drift diffusion model (DDM) [37, 42]- a four-parameter model fit to RT distributions corresponding to the two choices (left vs. right; Methods; Fig. 1-1D). We found that higher contrasts caused faster rates of evidence accumulation (drift rate, Fig. 1-1E), and also caused a systematic change in the starting point (Fig. 1-1G). These changes were complementary (Fig. 11I), thereby accounting for the lack of effect of target contrast on median RTs despite increase in drift rate (consistent with published reports [24])

The DDM analysis also yielded a quantitative estimate of $t_{\text {delay }}$, a parameter that represents the combined 'delay overheads' underlying a perceptual decision process. $T_{\text {delay }}$ accounts for: (a) the time taken for the sensory (visual) periphery to transduce and relay information to visual brain areas (i.e., neural response latency), as well as (b) the time taken for executing the motor response (i.e., motor execution delay). In our tasks, the latter corresponds to the time for the mouse to move its head (and body) to achieve the appropriate nose-touch. Notably, we found that stimulus contrast as well as size had no discernable effect on $t_{\text {delay }}$ (Fig. 1-1H. 2-way ANOVA, size: $\mathrm{p}=0.308$, contrast: $\mathrm{p}=0.523$; interaction: $\mathrm{p}=0.931$ ), and the average value of $\mathrm{t}_{\text {delay }}$ was $212 \mathrm{~ms}$.

Together, these results revealed a systematic effect of target contrast as well as size on discrimination accuracy, driven by an effect on perceptual sensitivity rather than response criterion. Additionally, they 
revealed that the combined delay overhead in the visual decision process was nearly constant across conditions, at $\sim 200 \mathrm{~ms}$ ( $\left.\mathrm{t}_{\text {delay }}\right)$.

\section{Effect of stimulus size and contrast on dynamics of visual decision-making: the sensory encoding stage}

To investigate the dynamics of visual perceptual decision-making, we adapted approaches from human studies and the examined the dependence of response accuracy on RT, i.e., the so-called 'conditional accuracy' function [9-11]. To this end, we first pooled trials across the different trial conditions ( 3 sizes $\mathrm{x}$ 7 contrasts) from all mice $(n=8)$, sorted them based on RT, and plotted conditional accuracy for each RT bin (100ms; Fig. 2A-orange dots; Methods). We found that for responses with RT less than $500 \mathrm{~ms}$, conditional accuracy improved for longer RT (Pearson's $\rho=0.99, p=0.02$ ), consistent with the classic 'speed-accuracy tradeoff' [34]. For responses with RT greater than $500 \mathrm{~ms}$ and up to $3 \mathrm{~s}$, the allowed duration for responses, conditional accuracy plateaued, and was independent of RT (Pearson's $\rho=0.33$, $\mathrm{p}=0.11$ ). Drawing upon arguments from human behavioral studies, we reasoned that the initial transient stage of the conditional accuracy function reflects the process of sensory encoding: during it, slower responses allow more sensory evidence to be acquired, thereby improving conditional accuracy upto a peak value reflecting the completion of sensory encoding [29-32].

We next quantified the dynamics of sensory encoding by fitting the conditional accuracy data with an asymptotic function (Fig. 2A - orange curve) [9-11], and estimating three key metrics: (1) the peak conditional accuracy $\left(a_{\text {peak }}\right),(2)$ the timepoint at which conditional accuracy just exceeded $50 \%$ (chance) performance $\left(t_{50}\right.$; Methods), and (3) the timepoint at which conditional accuracy reached its peak $\left(t_{\text {peak }}\right.$; Methods).

To examine the effect of stimulus size on sensory encoding dynamics, we fit trials of different stimulus sizes separately (Fig. 2B), and estimated the key metrics in each case (Methods; all contrasts included, all mice). We found that the peak conditional accuracy was significantly modulated by stimulus size (Fig.2Cleft; $a_{\text {peak }}$ : size $25^{\circ}$, mean \pm s.d. $=81.3 \pm 1.2 \%$; size $35^{\circ}=88.0 \pm 0.7 \%$; size $45^{\circ}=92.4 \pm 0.9 \%$; $*$, p $<0.05$, permutation tests with HBMC correction). The time to exceed chance performance and the time to reach peak accuracy, however, were not $\left(\mathrm{t}_{50}\right.$, Fig. $2 \mathrm{C}$-middle, size $25^{\circ}=190 \pm 31 \mathrm{~ms}$, size $35^{\circ}=221 \pm 14 \mathrm{~ms}$, size $45^{\circ}=193 \pm 20 \mathrm{~ms} ; \mathrm{t}_{\text {peak }}$, Fig. $2 \mathrm{C}$-right, size $25^{\circ}=491 \pm 56 \mathrm{~ms}$, size $35^{\circ}=461 \pm 22 \mathrm{~ms}$, size $45^{\circ}=467 \pm 26 \mathrm{~ms}$ ).

To examine the effect of stimulus contrast on sensory encoding dynamics (Fig. 2D), we divided trials (all sizes included, all mice) into two subsets: (1) trials with target contrast $\leq 54 \%$ ('low-contrast'), and (2) trials with target contrast $>54 \%$ ('high-contrast'; Methods). We fit each subset separately and found that the peak conditional accuracy was significantly modulated by stimulus contrast (Fig.2E-left; $a_{\text {peak }}$ : low-contrast $=84.6 \pm 0.9 \%$; high-contrast $=89.5 \pm 0.6 \%, \mathrm{p}<0.001$, permutation test). There was no significant effect of stimulus contrast on $t_{50}$ (Fig. 2E-middle, low-contrast $=213 \pm 20 \mathrm{~ms}$; high-contrast $=207 \pm 12 \mathrm{~ms}, \mathrm{p}=0.747$, permutation test), but the time to reach peak accuracy was significantly modulated (Fig.2E-right; $t_{\text {peak: }}$ lowcontrast $=532 \pm 30 \mathrm{~ms}$; high-contrast $=412 \pm 17 \mathrm{~ms}, \mathrm{p}<0.001$, permutation test). The shorter $\mathrm{t}_{\text {peak }}$ despite higher $\mathrm{a}_{\text {peak }}$ at higher contrasts indicated that the rate of sensory encoding was faster for higher contrast stimuli.

The findings that contrast and size alter $a_{\text {peak }}$ (and $t_{\text {peak }}$ ) demonstrate the causal role of stimulus features in controlling fundamental properties of sensory encoding dynamics. Additionally, because $t_{\text {peak }}$ represents the window for sensory encoding combined with the delay 'overheads', we estimated the duration of just the sensory encoding stage (temporal integration window) as $t_{\text {peak }}-t_{\text {delay, }}$ varying between $212 \mathrm{~ms}$ (412 ms $200 \mathrm{~ms}$; high contrast) and $332 \mathrm{~ms}$ (532 ms -200 ms, low contrast) across conditions.

Thus, conditional accuracy analysis revealed the presence of a sensory encoding stage in mouse visual perceptual dynamics, governed by the 'speed-accuracy tradeoff', and lasting up to $332 \mathrm{~ms}$. 

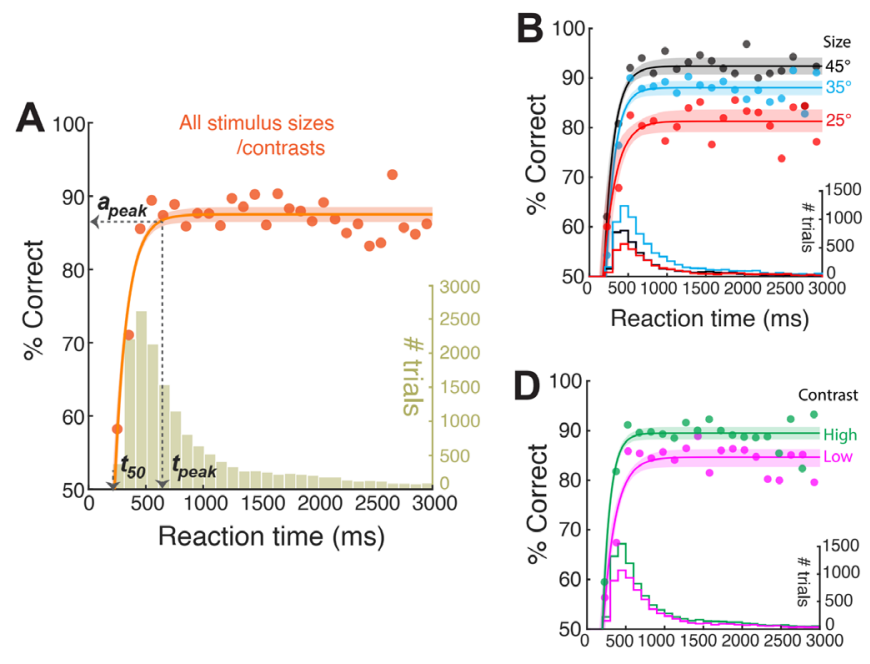
Reaction time (ms)
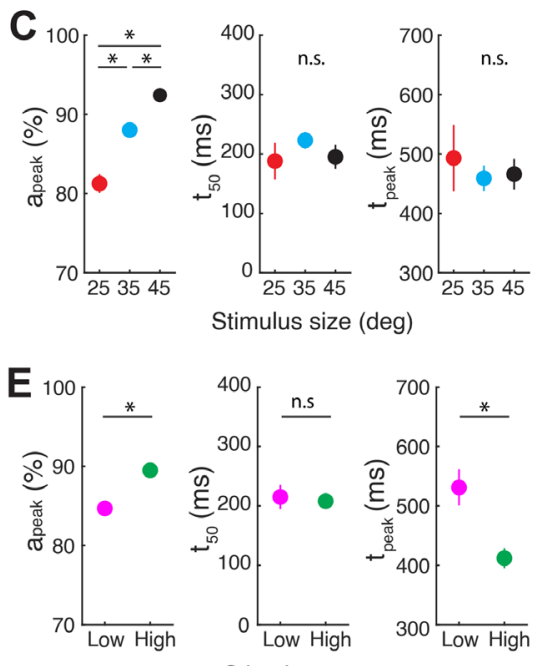

Stimulus contrast

Figure 2. Stimulus contrast and size modulate the sensory encoding stage of the conditional accuracy function (CAF). (A) Plot of accuracy as a function of RT bins (conditional accuracy) using same dataset as Fig. 1. Orange dots: Data pooled across all stimulus sizes and contrast ratios, $n=8$ mice; RT bin size $=100 \mathrm{~ms}$. Orange curve: Conditional accuracy function, CAF (best-fit rising asymptotic function; Methods); light orange shading: $95 \%$ CI of the fit (Methods). Indicated are three key parameters $\left(a_{\text {peak }}, t_{50}\right.$ and $\left.t_{\text {peak }}\right)$ describing the sensory encoding stage of the CAF the initial period during which accuracy improves for longer RT values, exhibiting a tradeoff between speed and accuracy (see text; Methods). Peak accuracy $\left(\mathrm{a}_{\text {peak }}\right)$ : mean \pm s.d. $=87.5 \pm 0.5 \%$; time to reach peak accuracy $\left(\mathrm{t}_{\text {peak }}\right): 462$ $\pm 13 \mathrm{~ms}$; time at which accuracy just exceeds $50 \%\left(\mathrm{t}_{50}\right): 236 \pm 10 \mathrm{~ms}$. Gold histogram: RT distribution (y-axis on the right). The overall response accuracy for a particular stimulus condition is the dot product of the CAF and the RT distribution. (B) CAFs for targets of various sizes (black: $45^{\circ}$; blue: $35^{\circ}$; red: $25^{\circ}$ ); conventions as in A. (C) Plots of key CAF parameters for different target sizes. Left panel: $a_{\text {peak }}$; middle panel: $t_{\text {peak }}$; right panel: $t_{50}$. Data show mean \pm s.t.d of distribution of bootstrapped estimates (Methods). '*' ('n.s.'): $\mathrm{p}<0.05(\mathrm{p}>0.05)$, paired permutation tests followed by HBMC correction (Methods). $a_{\text {peak: }}$ p $<0.001\left(25^{\circ}\right.$ vs. $\left.35^{\circ}\right), p<0.001\left(35^{\circ}\right.$ vs. $\left.45^{\circ}\right), p<0.001\left(25^{\circ}\right.$ vs. $\left.45^{\circ}\right)$; $\mathrm{t}_{\text {peak: }}$ : $=0.398\left(25^{\circ}\right.$ vs. $\left.35^{\circ}\right), \mathrm{p}=0.827\left(35^{\circ}\right.$ vs. $\left.45^{\circ}\right), \mathrm{p}=0.576\left(25^{\circ}\right.$ vs. $\left.45^{\circ}\right) ; \mathrm{t}_{50}: \mathrm{p}=0.226\left(25^{\circ}\right.$ vs. $\left.35^{\circ}\right), \mathrm{p}=0.127\left(35^{\circ}\right.$ vs. $\left.45^{\circ}\right), p=0.918\left(25^{\circ}\right.$ vs. $\left.45^{\circ}\right)$. (D) CAFs for targets of different contrast conditions (magenta: 'low' contrast - first three contrast ratio levels from Fig. 1C; green: 'high' contrast - last four contrast ratio levels; Methods); conventions as in A. (E) Plots of key CAF parameters for different contrast conditions; conventions and statistical methods as in C. $\mathrm{a}_{\text {peak }}: \mathrm{p}<0.001$ (low vs. high contrast conditions); $\mathrm{t}_{\text {peak }}: \mathrm{p}<0.001 ; \mathrm{t}_{50}: \mathrm{p}=0.747$.

\section{Stimulus duration and the dynamics of visual decision-making: the memory-dependent stage}

Following the sensory encoding stage, the next stage in the timecourse of perceptual decisions identified in human studies is one that reflects completion of sensory encoding and the availability of a fully constructed internal representation of the target stimulus for guiding behavior [43]. Consequently, during this so-called internal or 'short-term memory' (STM)-dependent stage, longer reaction times and additional sampling do not produce improvements in accuracy [30]. Additionally, during this stage, a drop in accuracy is observed once stimulus input is removed - attributed typically to decay of information in STM process [33-36].

In the experiments so far, following the sensory encoding stage (ending at $t_{\text {peak }}=532 \mathrm{~ms}$; Fig. 2A), we observed no further increase in performance with longer RTs, consistent with human studies. Additionally, we also observed that the performance plateaued and did not exhibit a decay, consistent with the target stimulus being present throughout the response window of 3s (Fig. 2A, B, D). 
To test for the existence of the second 'decaying' stage in mouse visual decision dynamics, we performed an experiment in which we shortened the stimulus duration systematically from $3 \mathrm{~s}$. This allowed us to examine decision behavior following stimulus offset. We reasoned that for trials in which mice initiated responses after the stimulus disappeared and, additionally, after the sensory encoding stage was also complete, a decline of conditional accuracy with longer RTs would reflect the reliance of mice on STM information for the production of correct responses [44-48]. In this experiment, stimulus size and contrast were maintained fixed at $25^{\circ}$ and $98 \%$, respectively.

As a first step in the analysis, we examined overall behavioral performance at different stimulus durations and found that it was significantly modulated (Fig.3A, one-way ANOVA, p=0.047), with accuracy decreasing with decrease in stimulus duration (Pearson's $\rho=0.74, p=0.01$ ). This effect was driven by a commensurate effect on perceptual sensitivity (Fig.3B, filled data; one-way ANOVA, $\mathrm{p}=0.001$; Pearson's $\rho=0.74, p=0.01$ ) but not decision criterion (Fig. $3 \mathrm{~B}$, hollow data; one-way ANOVA, $p=0.802$ ). There was also a trend of decreased RT as the stimulus duration decreased (Fig.3C, one-way ANOVA, p=0.133; Pearson's $\rho=0.86, \mathrm{p}<0.001)$. These results revealed, additionally, that the shortest stimulus duration needed for mice to be able to discriminate above chance was less than $100 \mathrm{~ms}$ - the smallest duration tested (Fig. $3 \mathrm{C})$.
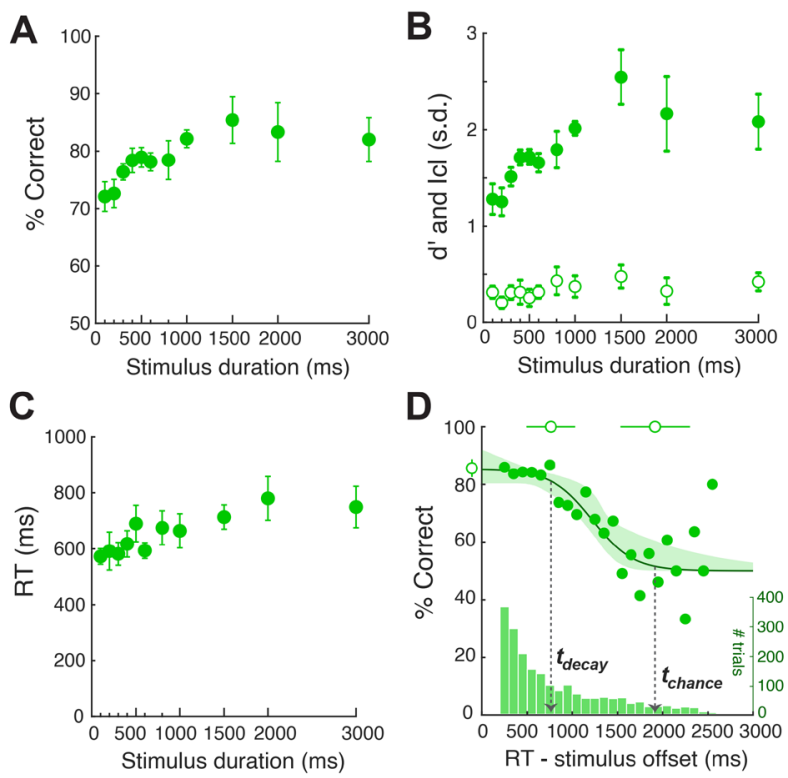

Figure 3. Stimulus duration and the memory-dependent stage of the conditional accuracy function.

(A) Psychometric plot of discrimination accuracy against stimulus duration. Data: mean \pm s.e.m; $\mathrm{n}=6$ mice. 1-way ANOVA; $p=0.047$. Stimulus size $=25^{\circ}$; contrast $=98 \%$. (B) Plot of discrimination sensitivity (d', filled data, $p=0.001$, 1-way ANOVA) and criteria ( $|\mathrm{c}|$, hollow data, $\mathrm{p}=0.802$, 1-way ANOVA) against stimulus duration. (C) Plot of median reaction time (RT) against stimulus duration. 1-way ANOVA; $\mathrm{p}=0.133$. (D) Plot of the conditional accuracy (solid data) as a function of RT bins relative to stimulus offset. Only trials in which the stimulus was longer than $332 \mathrm{~ms}$ (to ensure full sensory encoding; see text), and in which the response was initiated after the stimulus disappeared (i.e., with RT $\geq$ stimulus duration $+200 \mathrm{~ms}$; see text) were included (Methods). Curve and shading: best-fit sigmoid function and $95 \%$ C.I. Hollow data: bootstrapped estimates of the peak accuracy $\left(\mathrm{a}_{\text {peak }}\right.$, mean \pm s.t.d. $\left.=85.6 \pm 3.1 \%\right)$, $\mathrm{t}_{\text {decay }}(762$ $\pm 271 \mathrm{~ms})$ and $\mathrm{t}_{\text {chance }}(1916 \pm 385 \mathrm{~ms})$. Histogram: RT distribution (y axis on the right).

\section{See also Fig. 3-1.}

Next, to examine decision dynamics, we constructed the conditional accuracy function. As motivated above, we wished to include only those trials on which mice initiated responses after the stimulus disappeared, and also after the sensory encoding stage could have been completed. Therefore, we included only the trials on which the reaction time was longer than stimulus duration $+200 \mathrm{~ms}$ (estimate of $t_{\text {delay }}$ from Fig. S1G), and 
on which the stimulus was presented for longer than $332 \mathrm{~ms}$ (estimate of the duration of the sensory encoding stage from Fig. 2A). We aligned these trials to stimulus offset, and computed the conditional accuracy. We observed the classic decay in conditional accuracy with longer RTs (Fig. 3D).

To quantify the time course of the decay, we fit the conditional accuracy data with a sigmoidal function, and estimated three key metrics (Fig. 3D). First, the peak performance, $a_{\text {peak, }}$, was $85.6 \pm 3.1 \%$, comparable to the asymptotic level of Figure 2A, thereby supporting that sensory encoding is, indeed, complete on these trials. Second, the time point at which the conditional accuracy started to decline, $t_{\text {decay }}$, was $\sim 750 \mathrm{~ms}$ (762 $\pm 271 \mathrm{~ms}$ ) after stimulus offset. Third, the first timepoint at which the discrimination accuracy dropped to a level indistinguishable from the chance, $t_{\text {chance, }}$ was $\sim 1900$ (1916 $\pm 385 \mathrm{~ms}$ ) after stimulus offset (Methods).

Thus, similar to human studies, our results revealed a second stage in mouse visual perceptual dynamics in which, following completion of sensory encoding, performance after stimulus offset decreases gradually with reaction time, consistent with decay of information about the stimulus maintained in STM (see also Discussion). They revealed $1700 \mathrm{~ms}$ ( $1900 \mathrm{~ms}$ minus the $200 \mathrm{~ms}$ of $\mathrm{t}_{\text {delay }}$ ) as an estimate of the duration after stimulus offset beyond which information for correct responding is no longer available to the animals.

\section{Task-relevant foil ('flanker') modulates the sensory encoding stage of the conditional accuracy function}

The sensory context in which the perceptual target is presented is well known to effectively modulate animals' performance [49-51]. For instance, as demonstrated in the classic flanker task in humans, the cooccurrence of a foil stimulus with conflicting information can interfere with perceptual performance [52, 53]. Recently, similar results were demonstrated in mice using a touchscreen version of the flanker task [24]. In this task (Fig. 4A), a target grating (always presented at the lower location) was accompanied by a flanker grating at the upper location with either orthogonal orientation ('incongruent' flanker) or same orientation ('congruent' flanker). Compared to the presence of a congruent flanker, the 'incongruent' flanker significantly impaired discrimination accuracy (Fig. 4B-left; $\mathrm{p}<0.001$, signed-rank test. re-plotted based on data from [24]; Methods) and median RT (Fig. 4B-right, $\mathrm{p}=0.019$, signed-rank test). Here, we analyzed that dataset with the conditional accuracy analysis to investigate whether an incongruent flanker affected the sensory encoding stage or the STM-dependent stage of perceptual dynamics. The stimuli in this task were presented for $1 \mathrm{~s}$ and the response window was $3 \mathrm{~s}$ as before. 
A

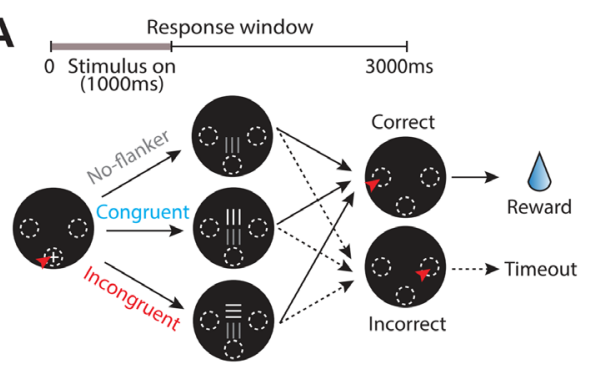

B

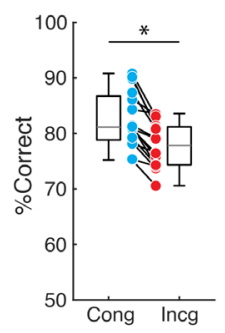

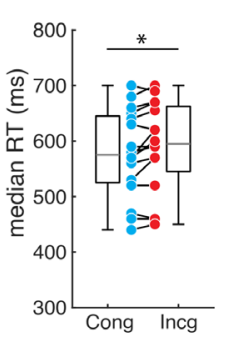

C

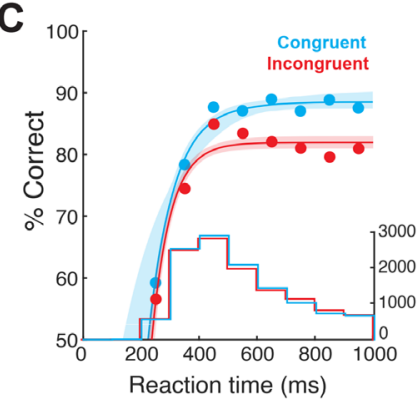

E

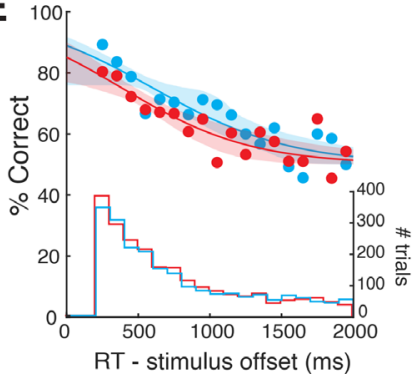

D

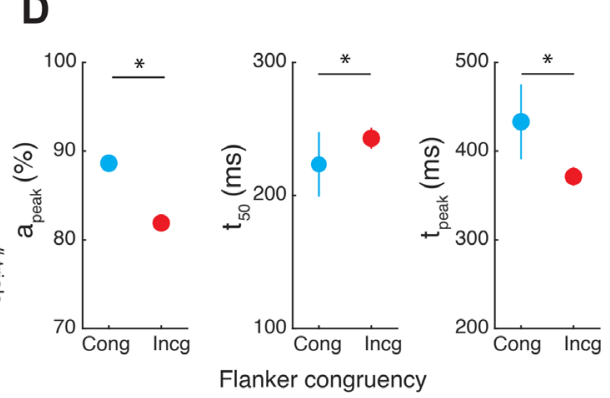

F

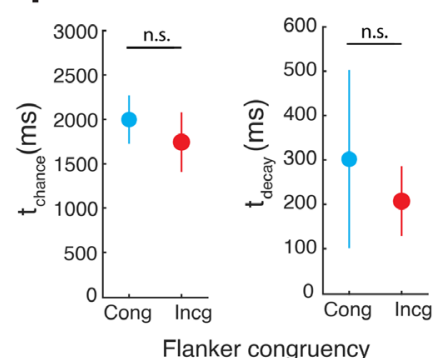

Figure 4. Incongruent flanker modulates the sensory encoding stage of the conditional accuracy function (CAF). (A) Schematic of the flanker task; target grating is always presented at the lower location; a second 'flanker' grating (orthogonal orientation - incongruent flanker, or same orientation - congruent flanker) is presented simultaneously, and always at the upper location; contrast of flanker is systematically varied (adapted from [24]). All other conventions as in Fig. 1. Plots represent results from new analyses applied to published data [24] after collapsing across all flanker contrasts (Methods).

(B) Left panel: Comparison of performance between trials with incongruent vs. congruent flanker. $p<0.001$, signed rank test. Right panel: Comparison of median RT between trials with incongruent vs. congruent flanker. $\mathrm{p}=0.019$, signed rank test. Data re-analyzed from You et al [24]; each line represents data from one mouse. (C) CAFs of the sensory encoding stage; data correspond to trials with RT < stimulus offset, i.e., $1000 \mathrm{~ms}$. Blue: trials with congruent flanker; red: trials with incongruent flanker; histograms; RT distributions.

(D) Plots of key parameters of CAFs (sensory encoding stage) for trials with congruent vs. incongruent flanker; $a_{\text {peak }}$ (left), $t_{50}$ (middle), and $t_{\text {peak }}$ (right). Data show mean \pm s.t.d of distribution of bootstrapped estimates. '*' ('n.s.'): $p<0.05$ ( $>0.05$ ), permutation tests followed by HBMC correction, congruent vs. incongruent flanker conditions (Methods). $a_{\text {peak }}$ : $p<0.001 ; \mathrm{t}_{50}$ : $\mathrm{p}=0.022 ; \mathrm{t}_{\text {peak }}$ : $\mathrm{p}=0.01$.

(E) CAFs of the STM-dependent stage; data correspond to trials with RT > stimulus offset (1000 ms) $+t_{\text {delay }}(200 \mathrm{~ms}$; see text); aligned to stimulus offset. Blue: trials with congruent flanker; red: trials with incongruent flanker.

(F) Plots of key parameters of CAFs (VSTM-dependent stage) for trials with congruent vs. incongruent flanker; $t_{\text {chance }}$ (left) and $t_{\text {decay }}$ (right). Conventions and statistical methods as in D. $t_{\text {chance: }} p=0.426 ; t_{\text {decay }}: p=0.313$.

We investigated the effect of an incongruent flanker on perceptual dynamics by first pooling trials from all mice into two groups based on their flanker congruency, and sorted the trials based on their RT. To investigate the sensory encoding stage, we followed the approach used in Figure 2 and selected the trials on which mice responded before the stimulus ended (RT $<1000 \mathrm{~ms})$, and aligned them to stimulus onset. 
Separately, to investigate the STM -dependent stage, we followed the approach in Figure 3 and selected the trials on which responses were initiated after the stimulus disappeared (i.e, with $\mathrm{RT}-\mathrm{t}_{\text {delay }}>1000 \mathrm{~ms}$ ), and thereby, by default, also after time needed for sensory encoding to be completed (i.e, $\mathrm{RT}-\mathrm{t}_{\text {delay }}>332 \mathrm{~ms}$ ), and aligned them to stimulus offset. ( $t_{\text {delay }}$ was taken to be $200 \mathrm{~ms}$ from Fig. 2 and 3.) (Performing a DDM on the RT distributions from this dataset revealed that $t_{\text {delay }}$ was $\sim 200 \mathrm{~ms}$, not different from the estimates in the previous experiments (mean $t_{\text {delay }}=205 \mathrm{~ms}$ for congruent trials, and $209 \mathrm{~ms}$ for incongruent trials))

We found that in the sensory encoding stage (Fig. 4CD), the peak conditional accuracy for incongruent trials was significantly lower than that of congruent trials (Fig. 4D-left; congruent $=88.6 \pm 0.8 \%$, incongruent $=81.9 \pm 0.5 \% ; \mathrm{p}<0.001$, permutation test), and the time at which performance just exceeded the $50 \%$ (chance) level was longer for incongruent trials (Fig. 4D-middle; $t_{50}$ : congruent $=223 \pm 24 \mathrm{~ms}$; incongruent $=243 \pm 8 \mathrm{~ms} \mathrm{p}=0.022$, permutation test) - both indicate that the presence of incongruent flanker interfered with the sensory encoding of the target stimulus. The time to reach peak accuracy was, however, shorter for incongruent trials (Fig. 4D-right; $t_{\text {peak }}$ : congruent $=433 \pm 42 \mathrm{~ms}$; incongruent $=371 \pm 11 \mathrm{~ms}$; $\mathrm{p}=0.01$, permutation test), consistent with the lower $a_{\text {peak }}$ (Fig. 4D-left).

By contrast, there was no effect of flanker congruency on the time course of decay of conditional accuracy following stimulus offset. The time at which conditional accuracy dropped to chance was not different between congruent and incongruent flanker trials (Fig. 4EF; $t_{\text {chance }}$ : congruent $=1998 \pm 274 \mathrm{~ms}$; incongruent $=1744 \pm 337 \mathrm{~ms}, \mathrm{p}=0.426$, permutation test), nor on the time at which conditional accuracy dropped just below $a_{\text {peak }}\left(\mathrm{t}_{\text {decay }}\right.$ : congruent $=302 \pm 201 \mathrm{~ms}$; incongruent $=207 \pm 79 \mathrm{~ms}, \mathrm{p}=0.313$, permutation test $)$.

In sum, we found that the interference in performance due to the incongruent flanker impacted the sensory encoding stage $\left(a_{\text {peak}}\right.$; as if weakening the target $\left.[54,55]\right)$, but not the STM -dependent stage, of mouse visual decision dynamics.

\section{Stimulus onset delay modulates RT distribution but not the conditional accuracy function}

In investigating behavioral performance, overall decision accuracy can be decomposed into two components - the conditional accuracy function and RT distribution (Fig. 2A); the dot product of these two quantities yields overall accuracy. Our manipulations, thus far, produced changes in the conditional accuracy function predominantly. We wondered whether task parameters could alter RT distribution and possibly do so without affecting conditional accuracy function. To test this, we added a delay between trial initiation and target onset (i.e., stimulus onset delay) in the single stimulus discrimination task. We reasoned that the extent to which mice are able to adaptively withhold responding could impact the RT distribution.

We found adding a stimulus onset delay does alter the RT distribution of mice (Fig. 5A-left; RT computed w.r.to trial initiation). The median RTs (relative to trial initiation) increased with delay (Fig. 5A-right; Pearson's $\rho=0.999, p=0.035$ ). This indicated that mice were able to sense the delayed onset of stimulus and thereby withhold their responses. Nonetheless, mice were unable to do so for the full duration required: the average increase in median RT was smaller than the increase in delay (Fig. 5A-right; average $\Delta$ median $\mathrm{RT}=36 \mathrm{~ms}$ for $100 \mathrm{~ms}$ delay, and $79 \mathrm{~ms}$ for $200 \mathrm{~ms}$ delay). This increase in median RT at longer delays was accompanied by a trend towards lower decision accuracy (Fig. 5B. Pearson's $\rho=0.988, p=0.098$ ).

By contrast, conditional accuracy analysis revealed no effect of stimulus onset delay either on the sensory encoding stage (Fig. 5C and S2D; $a_{\text {peak }}$ : no delay $=88.3 \pm 2.0 \%$; delay $=83.3 \pm 3.6 \% ; \mathrm{p}=0.228$, permutation test; $\mathrm{t}_{50}$ : no delay $=209 \pm 40 \mathrm{~ms}$; delay $=176 \pm 31 \mathrm{~ms} ; \mathrm{p}=0.652$, permutation test; $\mathrm{t}_{\text {peak }}$ : no delay $=485 \pm 36$ $\mathrm{ms}$; delay $=442 \pm 73 \mathrm{~ms} ; \mathrm{p}=0.473$, permutation test), or on the STM-dependent stage (Fig. 5D and S2E; $t_{\text {chance }}$ : no delay $=1581 \pm 277 \mathrm{~ms}$; delay $=2035 \pm 444 \mathrm{~ms} ; \mathrm{p}=0.261$, permutation test; $\mathrm{t}_{\text {decay: }}$ no delay $=919$ $\pm 226 \mathrm{~ms}$; delay $=543 \pm 277 \mathrm{~ms} ; \mathrm{p}=0.165$, permutation test). 
Taken together, our results from varying the stimulus onset delay show that changes in RT distribution (and/or decision accuracy) are not necessarily accompanied by changes in the conditional accuracy function, which implies that the underlying sensory processes could remain unchanged. The observed trend of decreased accuracy was accounted by the fact that with a delay, there were more responses initiated before the sensory encoding was complete, or even before the stimulus was presented (i.e., 'impulsive' responses) (Fig.5C). To quantify such impulsivity, we propose an 'impulsivity index' (ImpI): motivated by the observation that mice withheld responses at longer delays consistently for $\sim 40 \mathrm{~ms}$ for every $100 \mathrm{~ms}$ of delay (median $\mathrm{RT}_{\text {delay }=100}-$ median $\mathrm{RT}_{\text {delay }=0}=36 \mathrm{~ms}$; median $\mathrm{RT}_{\text {delay }=200}$ - median $\mathrm{RT}_{\text {delay }=100}=43 \mathrm{~ms}$ ), we therefore, defined $\operatorname{ImpI}=1-$ average (duration for which mice withhold responses /duration of the delay). Higher positive values of this index indicate greater impulsivity, with ImpI $=1$ indicating a complete inability to withhold responding in the face of stimulus delays (insensitivity to delays, or 'maximally' impulsive). In the case of our mice, ImpI is $\sim 0.6$.
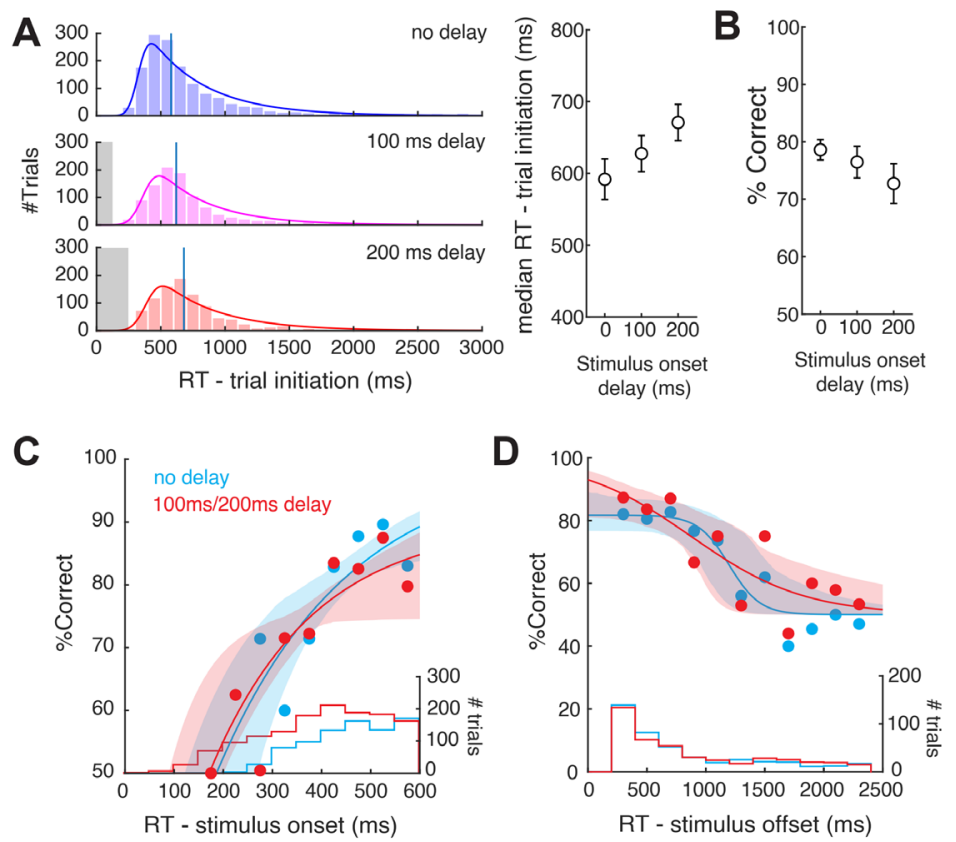

Figure 5. Stimulus onset delay modulates RT distribution but not the conditional accuracy function (A) Left: RT distributions at different stimulus onset delays (shown as shaded zones); plotted with response to time of trial initiation (for clarity). Right:Plot of median RT (w.r.to train initiation) against stimulus onset delay; $\mathrm{p}=0.138,1$ way ANOVA; Pearson's $\rho=0.99, \mathrm{p}=0.035$. (B) Plot of response accuracy against stimulus onset delay; $\mathrm{p}=0.337,1$ way ANOVA; $\rho=-0.988, p=0.098$. (C) Conditional accuracy functions of the sensory encoding stage; Blue: trials with no delay; red: trials with delay $=100$ and $200 \mathrm{~ms}$; shaded bands: bootstrap confidence intervals (95\%); confidence intervals overlap for the two datasets. Histograms: RT distributions. (D) Conditional accuracy functions of the STMdependent stage. Other conventions as in C.

See also Fig. 5-1.

\section{DISCUSSION}

Findings from this study have revealed two distinct stages in the temporal dynamics of visual perceptual decisions in mice, similar to those in humans. First, a sensory encoding stage that is subject to the speedaccuracy tradeoff, and then, a short-term memory dependent stage in which decision performance decays once the stimulus disappears. Our experiments also demonstrate that the two dissociable components of the dynamics of decision performance, namely the conditional accuracy function and the RT distribution, can 
be affected independently by experimental manipulations. Whereas experiments 1-3 revealed modulation of the conditional accuracy function (by stimulus size, contrast and presence of a foil) with minimal changes to the RT distribution, experiment 4 revealed modulation of the RT distribution (by stimulus onset asynchrony) without changes to the conditional accuracy function. Notably, our results yield quantitative estimates of fundamental psychophysical constants of visual perceptual decision-making in freely behaving mice. Together, this study establishes a quantitative platform for future work dissecting neural circuit underpinnings of the dynamics of visually guided decision-making in mice. The similarities in perceptual dynamics between mice and humans despite differences in the visual system suggest the presence of conserved principles underlying visual perceptual decision-making.

\section{Estimates of time constants of the dynamics of visual perceptual decision-making in mice}

Conditional accuracy analysis together with drift diffusion modeling and standard psychometric analyses yielded quantitative estimates of key parameters underlying the dynamics of visual decision-making in (freely behaving) mice. The duration of the sensory encoding stage (i.e., the window of temporal integration) was $\leq 332 \mathrm{~ms}$ across stimulus parameters and experimental conditions. Similarly, in humans, the internal representation of visual stimulus is thought to be constructed within the first 200-300 ms of stimulus presentation [29-32]; masking after that time does not impair performance. The duration of the fixed temporal overhead in the perceptual decision process, reflecting the combination of sensory (visual) latency and movement execution was $\sim 200 \mathrm{~ms}\left(\mathrm{t}_{\text {delay }}\right)$, and was largely unaffected by the tested stimulus parameters and experimental conditions. The shortest informative stimulus, i.e., the shortest stimulus duration which produced better than chance decision accuracy was $\sim 40 \mathrm{~ms}$ (estimated as $t_{50}-t_{\text {delay }}=236-200 \mathrm{~ms}$; Fig. $2 \mathrm{~A}$ ). Additionally, we developed a quantitative metric of impulsivity- one that depends on animals withholding responses until task-relevant information becomes available, rather than depending on withholding responses after all the information is available, or on the ability to stop a response that is underway [56]. For our freely behaving mice, this impulsivity index (ImpI, range 0 to 1 ) was 0.6 . (This inherent impulsivity can potentially be countered by directed/targeted training (as in [23]).

We also obtained an estimate of the duration of STM, i.e., the longest duration following stimulus offset for which mice could continue to perform above chance, as $1700 \mathrm{~ms}$. This constituted the period starting from stimulus offset to $200 \mathrm{~ms}$ before $t_{\text {chance }}$ (the last instant at which responses that are better than chance were initiated) (Fig. 3D; $t_{\text {chance }}-200 \mathrm{~ms}=\sim 1700 \mathrm{~ms}$ ). This duration of viability of the labile internal representation falls in the same range as has been reported from human studies [33, 34, 57]. This estimated duration of STM does not represent, necessarily, the duration for which visual stimulus information was maintained in STM. It could, also, represent maintenance of information about the motor response associated with the stimulus, or likely, a combination of the two.

Unlike STM, which refers to the retention of information even when it is not accessible from the environment, $\mathrm{WM}$ is thought of as 'STM+', referring additionally to the ability to manipulate this information and protect it from interference [58, 59]. From this perspective, our estimate of $1700 \mathrm{~ms}$ constitutes a lower bound for WM, which can potentially be lengthened with training. Indeed, tasks that require animals to hold information during enforced delay periods can cause the duration of the WM to be longer. For instance, in mice performing olfactory WM tasks, delay periods of up to $5 \mathrm{sec}$ have been reported [60]. Here, by allowing the natural evolution of the dynamics of decision-making to occur without an imposed delay period, we were able to estimate the 'intrinsic' duration of STM.

A potential factor that could confound our interpretation of the performance decay as being due to loss of information in STM is the attentional state of the animal. It is possible that the reduction in performance observed at longer RTs is due to trials in which mice did not pay attention to the stimulus, thereby reflecting lower accuracy (and longer RTs), rather than reflecting loss of information in STM. Our data from the flanker task (Fig. 4E) argue against this interpretation. In that experiment, we examined the time course of conditional accuracy after stimulus offset in two conditions in which we explicitly manipulated the attention 
of the animal (congruent vs. incongruent trials). We found that there was no difference in the decay of conditional accuracy between the two attentional conditions (Fig. 4E; red vs. blue). Thus, difference in attention across trials is unlikely to be the dominant factor accounting for the decay of conditional accuracy. Nonetheless, even if attention were a weak confounding factor, that would render our estimate of the duration of STM of $1700 \mathrm{~ms}$ as being, again, a lower bound.

\section{Estimates of other psychophysical constants, and operating range of stimulus features for visual perceptual decision-making in mice}

In addition to time constants of visual perceptual dynamics, this study yielded estimates of other psychophysical constants. The smallest stimulus and lowest contrast at which mice were able to discriminate orientation above chance were $25^{\circ}$ and $20 \%$, with mice performing at $>80 \%$ accuracy for most contrasts at that smallest size. Mice were also able to discriminate above chance at durations as short at 40 ms. All observed changes in discrimination accuracy were accompanied by changes in sensitivity rather than decision criterion, indicating that the manipulations all modulated aspects of the perceptual process. These findings that mice are able to discriminate visual stimuli in demanding sensory contexts suggest that the visual perceptual abilities of mice may be underrated.

The range of best discrimination performance of mice observed in our single target discrimination task (75$90 \%$, Fig. 1C) was lower than that of primates $(>90 \%)$ in similar tasks [61, 62]. Rather than reflecting fundamental differences in the decision processes, this is well accounted for by the lower visual acuity of mice, together with our use of 'small' stimuli (relative to those typically used in mouse vision studies [15, $16,18,20,63]$ ). Indeed, with larger grating stimuli, mice can perform very well with accuracy $>90 \%$ (Fig. S1J) $[18,64]$. The performance plateau of $93 \%$ for a stimulus size of $45^{\circ}$ (Fig. 1-1J), suggests that full-field stimuli may be effectively replaced by $45^{\circ}$ stimuli without appreciable loss in performance. In addition, the stimulus duration that was maximally effective was $1000 \mathrm{~ms}$, indicating that stimuli longer than $1000 \mathrm{~ms}$ may not be needed to test mouse behavior effectively in future single-stimulus discrimination tasks. (We note that although the conditional accuracy reaches a peak much earlier at $\sim 300 \mathrm{~ms}$, a longer stimulus is beneficial for overall accuracy due to the non-trivial fraction of trials that occupy the right half of the RT distribution, the consequence of overall accuracy being a dot product of the conditional accuracy function and RT distribution.)

At the highest contrasts, we observed a dip in performance from the plateau performance. Since the visual system adapts to the range of stimulus contrasts for best encoding [65], it is possible that the interleaved presentation of stimuli of various contrasts rendered the full-contrast stimulus unfavorable because of signal saturation [18]. Our data are directly consistent with this idea (Fig.1C vs. Fig. 1-1J).

\section{Optimal sensory sampling during visual perceptual decision-making in mice}

An intriguing observation across tasks was that mice responded with nearly constant RT (Fig. 1, 4, and 5; RT change $\leq 100 \mathrm{~ms}$, in contrast to the much larger change, $>500 \mathrm{~ms}$, seen in monkey perceptual decisionmaking tasks [66-68]). The conditional accuracy analysis offers a plausible account for this observation. As indicated by the conditional accuracy function, mouse response accuracy increased as RT increased until it reached a plateau at $t_{\text {peak. }}$. Therefore, an optimal strategy for mice would be to respond with RTs centered around $t_{\text {peak }}$ : responding earlier than $t_{\text {peak }}$ would sacrifice accuracy, while responding later than $t_{\text {peak }}$ would needlessly delay response (reducing the reward rate). Consistent with this expectation, their RT distribution was centered around $t_{\text {peak }}(500-600 \mathrm{~ms}$, Fig. 2ABD, 4C), indicating that mice responded at nearoptimal timing in terms of the speed-accuracy tradeoff.

\section{EXTENDED DATA}

Extended data (Fig. 1-1, 3-1, and 5-1) and legends are included. 


\section{REFERENCES}

1. Siegel, M., A.K. Engel, and T.H. Donner, Cortical network dynamics of perceptual decision-making in the human brain. Frontiers in human neuroscience, 2011. 5: p. 21.

2. $\quad$ Stanford, T.R., et al., Perceptual decision making in less than 30 milliseconds. Nature neuroscience, 2010. 13(3): p. 379.

3. Uchida, N., A. Kepecs, and Z.F. Mainen, Seeing at a glance, smelling in a whiff: rapid forms of perceptual decision making. Nature Reviews Neuroscience, 2006. 7(6): p. 485-491.

4. Wilming, N., et al., Large-scale dynamics of perceptual decision information across human cortex. Nature communications, 2020. 11(1): p. 1-14.

5. Steinemann, N.A., R.G. O'Connell, and S.P. Kelly, Decisions are expedited through multiple neural adjustments spanning the sensorimotor hierarchy. Nature communications, 2018. 9(1): p. 1-13.

6. Thura, D. and P. Cisek, Deliberation and commitment in the premotor and primary motor cortex during dynamic decision making. Neuron, 2014. 81(6): p. 1401-1416.

7. Zariwala, H.A., et al., The limits of deliberation in a perceptual decision task. Neuron, 2013. 78(2): p. 33951.

8. Yang, Y., et al., Millisecond-scale differences in neural activity in auditory cortex can drive decisions. Nature Precedings, 2008: p. 1-1.

9. Wickelgren, W.A., Speed-accuracy tradeoff and information processing dynamics. Acta psychologica, 1977. 41(1): p. 67-85.

10. McElree, B. and B.A. Dosher, Serial position and set size in short-term memory: the time course of recognition. Journal of Experimental Psychology: General, 1989. 118(4): p. 346.

11. Heitz, R.P., The speed-accuracy tradeoff: history, physiology, methodology, and behavior. Frontiers in neuroscience, 2014. 8: p. 150.

12. Huberman, A.D. and C.M. Niell, What can mice tell us about how vision works? Trends Neurosci, 2011. 34(9): p. 464-73.

13. Glickfeld, L.L., R.C. Reid, and M.L. Andermann, A mouse model of higher visual cortical function. Current opinion in neurobiology, 2014. 24: p. 28-33.

14. Seabrook, T.A., et al., Architecture, function, and assembly of the mouse visual system. Annual review of neuroscience, 2017. 40: p. 499-538.

15. Prusky, G.T., P.W. West, and R.M. Douglas, Behavioral assessment of visual acuity in mice and rats. Vision Res, 2000. 40(16): p. 2201-9.

16. Prusky, G.T. and R.M. Douglas, Characterization of mouse cortical spatial vision. Vision Res, 2004. 44(28): p. 3411-8.

17. Histed, M.H., L.A. Carvalho, and J.H. Maunsell, Psychophysical measurement of contrast sensitivity in the behaving mouse. J Neurophysiol, 2012. 107(3): p. 758-65.

18. Long, M., et al., Contrast-dependent orientation discrimination in the mouse. Sci Rep, 2015. 5: p. 15830.

19. Burgess, C.P., et al., High-Yield Methods for Accurate Two-Alternative Visual Psychophysics in HeadFixed Mice. Cell Rep, 2017. 20(10): p. 2513-2524.

20. Busse, L., et al., The detection of visual contrast in the behaving mouse. J Neurosci, 2011. 31(31): p. 11351-61.

21. Carandini, M. and A.K. Churchland, Probing perceptual decisions in rodents. Nature neuroscience, 2013. 16(7): p. 824.

22. Glickfeld, L.L., M.H. Histed, and J.H. Maunsell, Mouse primary visual cortex is used to detect both orientation and contrast changes. J Neurosci, 2013. 33(50): p. 19416-22.

23. Wang, L. and R.J. Krauzlis, Visual Selective Attention in Mice. Curr Biol, 2018. 28(5): p. 676-685.e4.

24. You, W.-K. and S.P. Mysore, Endogenous and exogenous control of visuospatial selective attention in freely behaving mice. Nature Communications, 2020. 11(1): p. 1-14.

25. Speed, A., et al., Spatial attention enhances network, cellular and subthreshold responses in mouse visual cortex. Nature communications, 2020. 11(1): p. 1-11.

26. Umino, Y., R. Pasquale, and E. Solessio, Visual temporal contrast sensitivity in the behaving mouse shares fundamental properties with human psychophysics. eNeuro, 2018. 5(4).

27. Nomura, Y., et al., Evaluation of critical flicker-fusion frequency measurement methods using a touchscreen-based visual temporal discrimination task in the behaving mouse. Neuroscience research, 2019. 148: p. 28-33.

28. Mar, A.C., et al., The touchscreen operant platform for assessing executive function in rats and mice. Nat Protoc, 2013. 8(10): p. 1985-2005. 
29. Shibuya, H. and C. Bundesen, Visual selection from multielement displays: measuring and modeling effects of exposure duration. Journal of Experimental Psychology: Human Perception and Performance, 1988. 14(4): p. 591.

30. Busey, T.A. and G.R. Loftus, Sensory and cognitive components of visual information acquisition. Psychological Review, 1994. 101(3): p. 446.

31. Vogel, E.K., G.F. Woodman, and S.J. Luck, The time course of consolidation in visual working memory. Journal of Experimental Psychology: Human Perception and Performance, 2006. 32(6): p. 1436.

32. Bays, P.M., et al., Temporal dynamics of encoding, storage, and reallocation of visual working memory. Journal of vision, 2011. 11(10): p. 6-6.

33. Posner, M.I. and S.W. Keele, Decay of visual information from a single letter. Science, 1967. 158(3797): p. 137-139.

34. Phillips, W. and A. Baddeley, Reaction time and short-term visual memory. Psychonomic Science, 1971. 22(2): p. 73-74.

35. Dick, A., Iconic memory and its relation to perceptual processing and other memory mechanisms. Perception \& Psychophysics, 1974. 16(3): p. 575-596.

36. Coltheart, M., Iconic memory and visible persistence. Perception \& psychophysics, 1980. 27(3): p. 183228.

37. Ratcliff, R., et al., Diffusion Decision Model: Current Issues and History. Trends Cogn Sci, 2016. 20(4): p. 260-281.

38. Guo, Z.V., et al., Procedures for behavioral experiments in head-fixed mice. PLoS One, 2014. 9(2): p. e88678.

39. Stanislaw, H. and N. Todorov, Calculation of signal detection theory measures. Behav Res Methods Instrum Comput, 1999. 31(1): p. 137-49.

40. Voss, A., J. Voss, and V. Lerche, Assessing cognitive processes with diffusion model analyses: a tutorial based on fast-dm-30. Front Psychol, 2015. 6: p. 336.

41. Voss, A., M. Nagler, and V. Lerche, Diffusion models in experimental psychology: a practical introduction. Exp Psychol, 2013. 60(6): p. 385-402.

42. $\quad$ Ratcliff, R., A theory of memory retrieval. Psychological Review, 1978. 85(2): p. 59-108.

43. Smith, P.L. and R. Ratcliff, An integrated theory of attention and decision making in visual signal detection. Psychol Rev, 2009. 116(2): p. 283-317.

44. Brown, J., Some tests of the decay theory of immediate memory. Quarterly Journal of Experimental Psychology, 1958. 10(1): p. 12-21.

45. Gold, J.M., et al., Visual memory decay is deterministic. Psychological Science, 2005. 16(10): p. 769-774.

46. Zhang, W. and S.J. Luck, Sudden death and gradual decay in visual working memory. Psychological science, 2009. 20(4): p. 423-428.

47. Barrouillet, P. and V. Camos, As time goes by: Temporal constraints in working memory. Current Directions in Psychological Science, 2012. 21(6): p. 413-419.

48. Ricker, T.J., E. Vergauwe, and N. Cowan, Decay theory of immediate memory: From Brown (1958) to today (2014). The Quarterly Journal of Experimental Psychology, 2016. 69(10): p. 1969-1995.

49. Whitney, D. and D.M. Levi, Visual crowding: a fundamental limit on conscious perception and object recognition. Trends Cogn Sci, 2011. 15(4): p. 160-8.

50. Meier, P., E. Flister, and P. Reinagel, Collinear features impair visual detection by rats. Journal of vision, 2011. 11(3): p. 22-22.

51. Miller, J., The flanker compatibility effect as a function of visual angle, attentional focus, visual transients, and perceptual load: a search for boundary conditions. Percept Psychophys, 1991. 49(3): p. 270-88.

52. Eriksen, B.A. and C.W. Eriksen, Effects of noise letters upon the identification of a target letter in a nonsearch task. Perception \& Psychophysics, 1974. 16(1): p. 143-149.

53. Fan, J., et al., Testing the efficiency and independence of attentional networks. J Cogn Neurosci, 2002. 14(3): p. 340-7.

54. Lee, J. and J.H. Maunsell, The effect of attention on neuronal responses to high and low contrast stimuli. Journal of neurophysiology, 2010. 104(2): p. 960-971.

55. Carrasco, M., S. Ling, and S. Read, Attention alters appearance. Nat Neurosci, 2004. 7(3): p. 308-13.

56. Dent, C.L. and A.R. Isles, An overview of measuring impulsive behavior in mice. Curr Protoc Mouse Biol, 2014. 4(2): p. 35-45.

57. Sperling, G., The information available in brief visual presentations. Psychological monographs: General and applied, 1960. 74(11): p. 1. 
58. Postle, B. and T. Pasternak, Short term and working memory. Encyclopedia of Neuroscience. San Diego, CA: Elsevier, 2009: p. 783-9.

59. Cowan, N., What are the differences between long-term, short-term, and working memory? Progress in brain research, 2008. 169: p. 323-338.

60. Liu, D., et al., Medial prefrontal activity during delay period contributes to learning of a working memory task. Science, 2014. 346(6208): p. 458-63.

61. Vazquez, P., M. Cano, and C. Acuna, Discrimination of line orientation in humans and monkeys. J Neurophysiol, 2000. 83(5): p. 2639-48.

62. Vogels, R. and G.A. Orban, How well do response changes of striate neurons signal differences in orientation: a study in the discriminating monkey. J Neurosci, 1990. 10(11): p. 3543-58.

63. Wong, A.A. and R.E. Brown, Visual detection, pattern discrimination and visual acuity in 14 strains of mice. Genes Brain Behav, 2006. 5(5): p. 389-403.

64. Andermann, M.L., A.M. Kerlin, and R.C. Reid, Chronic cellular imaging of mouse visual cortex during operant behavior and passive viewing. Front Cell Neurosci, 2010. 4: p. 3.

65. Ohzawa, I., G. Sclar, and R.D. Freeman, Contrast gain control in the cat visual cortex. Nature, 1982. 298(5871): p. 266-8.

66. Roitman, J.D. and M.N. Shadlen, Response of neurons in the lateral intraparietal area during a combined visual discrimination reaction time task. Journal of neuroscience, 2002. 22(21): p. 9475-9489.

67. Palmer, J., A.C. Huk, and M.N. Shadlen, The effect of stimulus strength on the speed and accuracy of a perceptual decision. Journal of vision, 2005. 5(5): p. 1-1.

68. Zylberberg, A., C.R. Fetsch, and M.N. Shadlen, The influence of evidence volatility on choice, reaction time and confidence in a perceptual decision. Elife, 2016. 5: p. e17688. 


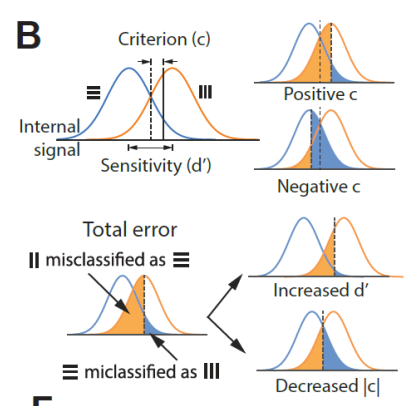

F
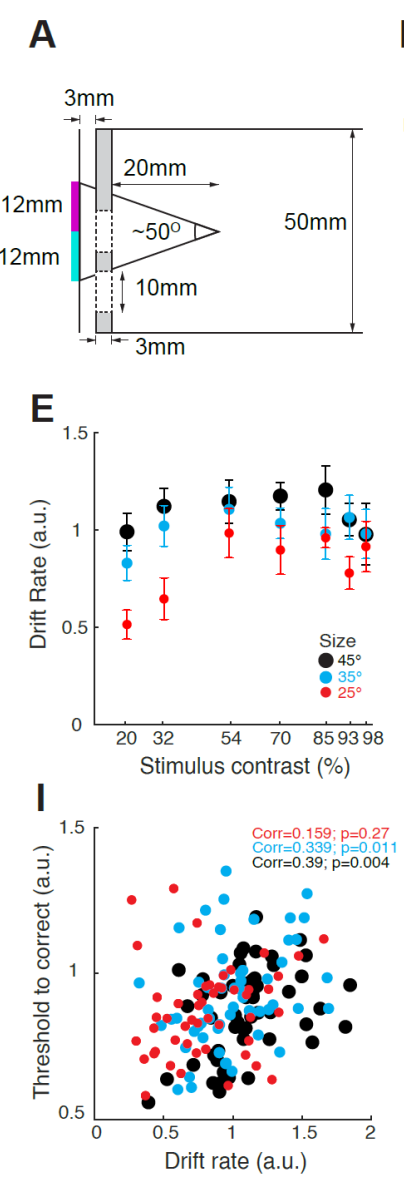

$\mathbf{J}$

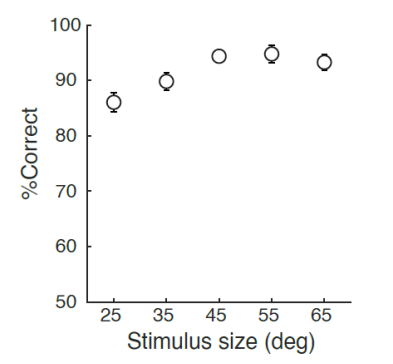

C
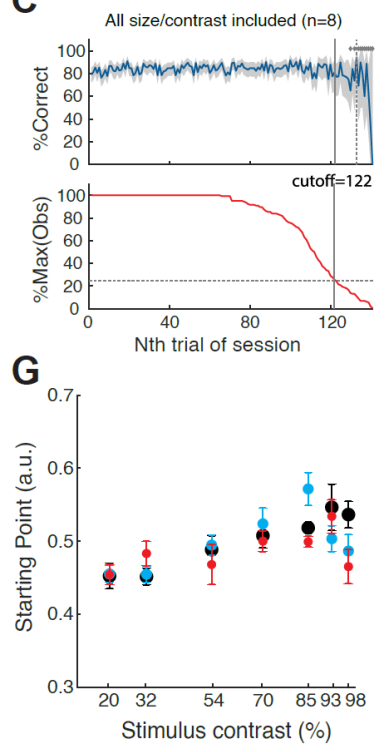
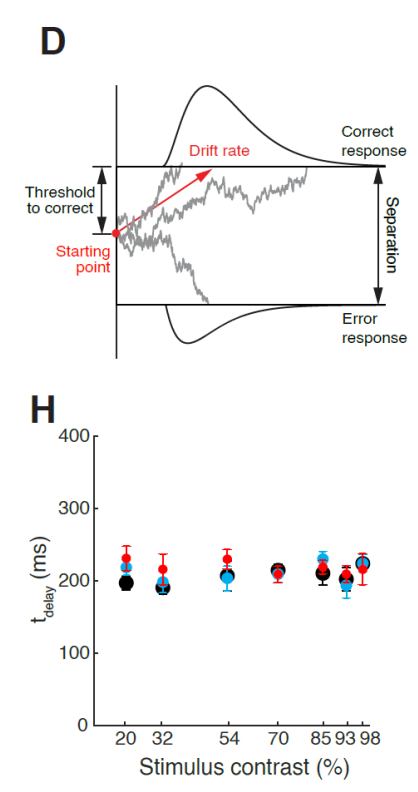

Figure 1-1. Extended data for stimulus contrast and size experiment; drift diffusion modeling.

(A) Lateral view of the schematic experimental setup showing the relative position of the touchscreen (leftmost vertical line), the plexiglass mask (grey-filled vertical bar), and the tube within which mice move (50 mm diameter); the plexiglass mask is positioned $3 \mathrm{~mm}$ in front of the touchscreen. Dashed lines indicate the central response hole (lower dashed lines), and left/right response holes (upper dashed lines; $10 \mathrm{~mm}$ diameter). For single-stimulus discrimination, the center of the stimulus is aligned with the center of left/right response holes in elevation, and with the central hole in azimuth (see Fig.1A). For experiments involving two stimulus locations (i.e., flanker task), the upper (magenta) and lower (cyan) locations of the stimulus are indicated as colored bars (see also Fig. 4A). The 60 pixels x 60 pixels $(12 \mathrm{~mm} \times 12 \mathrm{~mm})$ stimulus subtends a visual angle of $25^{\circ}$ when viewed from $20 \mathrm{~mm}$ front of the plexiglass mask.

(B) Schematic of the signal detection theory (SDT) analysis illustrating perceptual sensitivity (d') and criterion (c) calculations for 2-AFC task (Methods). Upper row; left: SDT hypothesizes that the internal representation of vertical and horizontal stimuli can be reduced (projected) to a one-dimensional decision axis, on which they form two overlapping distributions (due to noise). A decision is made based on a criterion set by each individual animal: a stimulus whose representation falls above (or below) the criterion is judged as vertical (or horizontal), producing the appropriate behavioral response. A decision criterion (c) of 0 , by definition, corresponds to optimal (unbiased) performance given the two distributions. For our 2-AFC task, we defined the decision criterion as the amount of deviation from an unbiased value for the following reason. Upper row; right: Because of the inherent symmetry of 2AFC task design, positive criterion would increase errors in classification of vertical targets, but also slightly decrease errors in classification of horizontal targets, producing a net reduction in overall accuracy. Similarly, a negative criterion would increase errors in classification of horizontal targets, but also slightly decrease errors in classification of vertical targets, again producing a net decrease in overall accuracy. Therefore, when the two distributions are similar, a negative as well as a positive criterion of the same magnitude will produce the same overall reduction in 
discrimination accuracy, but a criterion of smaller absolute value would signal an overall improvement in performance. For this reason, we used the absolute value of $\mathrm{c}(|\mathrm{c}|)$ to examine the effect of criterion change on response accuracy. Lower row: Based on theory, improved response accuracy can result from (1) increased d': when the two distributions become further separated; or (2) decreased $|\mathrm{c}|$ : when the decision criterion becomes less biased.

(C) Identification of trials towards the end of the $30 \mathrm{~min}$ behavioral sessions that corresponded to animals being poorly engaged in the task (Methods). Top panel: Time course of overall response accuracy across mice as a function of trial number within sessions. Accuracy obtained from trials pooled across all mice and sessions, and computed as a function of trial number within session (blue; Methods). Grey shading: bootstrapped estimates of the $95 \%$ confidence interval of the accuracy (gray; Methods). Diamonds on top: trials whose accuracy not significantly different from chance. Dashed vertical line: first trial at which the accuracy was not different from chance $(50 \%)$, and stayed indistinguishable from chance for $3 / 5$ of the next 5 trials (Methods). Data show increased variability and worse performance towards the end of sessions. Bottom panel: Number of actual observations across mice for each trial number, as a percentage of the maximal number of possible observations ( $\Sigma$ mice*sessions), plotted as a function of trial number within session (red). Solid vertical line: first trial at which the number of observations drops below $25 \%$. Data show drop in the number of observations available to reliably assess performance towards the end of sessions. Based on these data, all trials above 122 of each behavioral session of this experiment were dropped from analysis (Methods). Results in Fig. 1 are based on data from trials 1-121 from each behavioral session.

(D) Schematic diagram of the two-choice drift diffusion model. The model simulates a decision process from sensory stimulus presentation to the point of behavioral report, and attempts to account for the full distribution of observed RTs. It posits that upon stimulus presentation, sensory evidence flows in, causing a (hypothetical) decision variable to 'drift' either upwards (towards one choice boundary) or downwards (towards the other) depending on which choice the incoming evidence favors. Under uncertainty, the decision variable drifts in a stochastic (zig-zag) manner as sensory evidence accumulates, eventually crossing one of the decision boundaries and triggering the corresponding behavioral response. Here, we adopted a standard version of the model with four parameters: (i) drift rate, or the average rate of evidence accumulation, whose sign could be either positive (favoring response A) or negative (favoring response B); (ii) boundary separation, the distance by which the two decision boundaries are separated; (iii) starting point, which captures an initial bias towards one or the other choice (starting point $=0.5$ indicates an unbiased decision maker), and (iv) a non-decisional constant ( $\mathrm{t} 0$ ), which accounts for net delay due to sensory latency (before decisional process) and motor execution (after a decision has been made); not illustrated here. Also indicated here is 'thresholdto-correct response'.

(E-H) Plots of estimates of the four parameters of the drift diffusion model as a function of stimulus contrast (x-axis) and stimulus size (colors). (E) Drift rate; 2-way ANOVA, $\mathrm{p}=0.028$ (contrast), $\mathrm{p}<0.001$ (size), $\mathrm{p}=0.767$ (interaction). (F) Boundary separation; 2-way ANOVA, $\mathrm{p}=0.171$ (contrast), $\mathrm{p}=0.026$ (size), $\mathrm{p}=0.953$ (interaction). (G) Starting point; 2-way ANOVA, $\mathrm{p}<0.001$ (contrast), $\mathrm{p}=0.325$ (size), $\mathrm{p}=0.098$ (interaction). (H) tdelay; 2-way ANOVA, $\mathrm{p}=0.523$ (contrast), $\mathrm{p}=0.308$ (size), $\mathrm{p}=0.931$ (interaction).

(I) Scatter plot of drift-rate vs. threshold-to-correct response (indicated schematically in D) for different stimulus contrast. Each dot is data from a mouse at a particular contrast and size of the target; colors correspond to different sizes. Threshold-to-correct significantly correlated with drift rate (across contrasts) for stimulus size $=35^{\circ}(0.339$, $\mathrm{p}=0.11)$, and $45^{\circ}(0.39 ; \mathrm{p}=0.004)$.

(J) Discrimination accuracy as a function of stimulus size; $p=0.001$, 1-way ANOVA against stimulus size. 
A

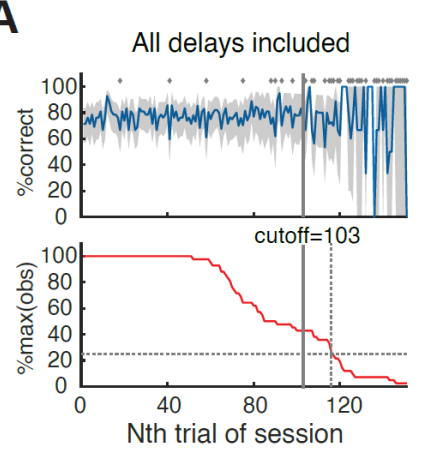

B

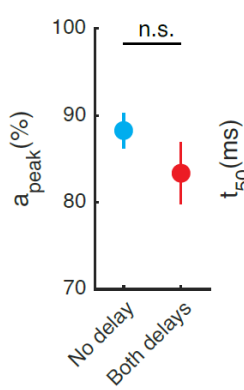

All duration included $(n=6)$

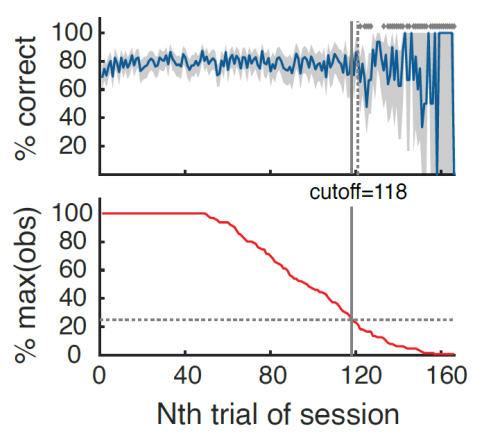

Figure 3-1. Extended data for stimulus duration experiment. Identification of trials towards the end of the $30 \mathrm{~min}$ behavioral sessions that corresponded to animals being poorly engaged in the task (Methods); conventions identical to those in Fig.S1C.

Figure 5-1. Extended data for stimulus onset delay experiment.

(A) Identification of trials towards the end of the $30 \mathrm{~min}$ behavioral sessions that corresponded to animals being poorly engaged in the task (Methods). All conventions are as in Fig.S1C. Based on these data, all trials above 103 of each behavioral session of this experiment were dropped from analysis. Results in Fig.5 are based on data from trials 1-102 from each behavioral session.

(B) Plots of key parameters of the conditional accuracy function for the sensory encoding stage of decision dynamics. Left panel: $a_{\text {peak }}$; middle panel: $t_{\text {peak }}$; right panel: $t_{50}$. Data show mean \pm s.t.d of distribution of bootstrapped estimates (Methods). '*' ('n.s.'): $\mathrm{p}<0.05$ (p $>0.05$ ), paired permutation tests followed by HBMC correction (Methods).

(C) Plots of key parameters of the conditional accuracy function for the STM-dependent stage of decision dynamics. Left panel: $t_{\text {chance }}$; right panel: $t_{\text {decay }}$. Data show mean \pm s.t.d of distribution of bootstrapped estimates (Methods). ' $*$ ' ('n.s.'): $\mathrm{p}<0.05$ ( $\mathrm{p}>0.05)$, paired permutation tests followed by HBMC correction (Methods). 